\title{
AC loss in large-scale superconducting cables
}

\author{
G.B.J. Mulder \\ Applied Superconductivity Centre, University of Twente, P.O. Box 217, 7500 AE Enschede, Netherlands
}

\begin{abstract}
A review is given of recent work on ac losses, carried out at our institute. The emphasis is on large-scale conductors for fusion applications, such as the 'cable-in-conduit' prototype conductors to be used for NET. Calculation methods for the ac losses are presented together with some experimental results.
\end{abstract}

\section{Introduction}

The ac losses in superconducting composites have been a subject of study for a long time, but nevertheless several problems remain to be solved, especially with respect to large-scale cables where the geometry of the cable plays an important role. Some of the aspects that will be considered here, are:

- presence of fields parallel with the conductor,

- coupling loss in composite conductors having various regions of stabilizer and/or superconductor,

- interstrand coupling loss in cables,

- influence of the reinforcement jacket on the loss,

- combined effect of transport current and alternating magnetic fields in different directions.

In order to calculate the overall loss in a complex superconducting coil system such as the NET fusion machine [1], the current scenario has to be divided in time steps during which $\dot{B}$ is almost constant. The magnetic field vectors $\boldsymbol{B}(t)$ and $\boldsymbol{B}(t+\Delta t)$ are calculated at regular positions in the coils and are decomposed in local components perpendicular and parallel with the cable. The key to this approach is to have suitable methods for determining the local energy loss from $B(t), \Delta B, \Delta t$, the orientation of $\Delta B$ with respect to the cable and the geometry of the cable. Then, the losses can be integrated over time and over the volume of the coils.

Tokamak fusion reactors are very interesting from the point of view of ac losses. The cabled conductors have a complicated geometry and they are subjected to

Correspondence to: Dr. G.B.J. Mulder, Philips Medical Systems Nederland, P.O. Box 10000, 5680 DA Best, The Netherlands. alternating and rotating magnetic field components in transverse and longitudinal directions (especially in the toroidal coils). The conductors of the poloidal as well as the toroidal system should be able to withstand the large field rates (up to $50 \mathrm{~T} / \mathrm{s}$ ) occurring during plasma disruptions, so the coupling loss has to be carefully considered. On the other hand, during regular operation of the fusion machine the fields rates are much lower (less than $1 \mathrm{~T} / \mathrm{s}$ ) so the hysteresis loss becomes relatively important.

\section{Filament hysteresis}

\subsection{Transverse field}

The hysteresis loss can be computed with the usual formulas if the magnetic field is purely perpendicular $[2,3]$. We can define the penetration field $B_{\mathrm{p} \perp}$, equal to $\mu_{0} j_{\mathrm{c}} d_{\mathrm{fil}} / \pi$ for round filaments. In addition, $M_{\mathrm{p} \perp}$ is defined as the magnetic moment per unit volume of a penetrated filament carrying $+j_{\mathrm{c}}$ in half of its cross section and $-j_{\mathrm{c}}$ in the other half. For round filaments $M_{\mathrm{p} \perp}$ is $-(2 / 3 \pi) j_{\mathrm{c}} d_{\mathrm{fil}} \operatorname{sign}(\Delta B)$. In large-scale applications the field variations are usually much larger than the penetration field $B_{\mathrm{p} \perp}$. In that case, the filaments remain fully penetrated and the power loss per unit volume of filament satisfies

$p_{\mathrm{h} \perp}=-M_{\mathrm{p} \perp} \dot{B}_{\perp}=\frac{2}{3 \pi} j_{\mathrm{c}} d_{\mathrm{fil}}\left|\dot{B}_{\perp}\right|$.

The presence of a transport current will reduce the filament magnetization by a factor of $1-\left(j_{\mathrm{s}} / j_{\mathrm{c}}\right)^{2}$, with $j_{\mathrm{S}}$ the current density averaged over the cross section. However, under ac conditions the filament has a dy- 
namic resistivity proportional to $\left|\dot{B}_{1}\right|$, therefore an extra loss $p_{\mathrm{t}}$ will appear when a transport current flows. The relation between $j_{\mathrm{s}}$ and the average electrical field along the filament $E_{\|}$can be approximated by [3]

$j_{\varsigma}= \begin{cases}j_{\mathrm{c}} E_{\|} / E^{*} & \text { if }\left|E_{\|}\right|<E^{*}, \\ j_{\mathrm{c}} \operatorname{sign}\left(E_{\|}\right) & \text {if }\left|E_{\|}\right| \geqslant E^{*},\end{cases}$

with $E^{*}=\frac{4}{3 \pi} d_{\mathrm{fil}}\left|\dot{B}_{\perp}\right|$. The total dissipation is calculated as $j_{s} E_{\|}-M \dot{B}$, so for unsaturated filaments with $j_{s}<j_{\text {c }}$ we obtain

$p_{\mathrm{h} \perp}+p_{\mathrm{t}} \cong \frac{2}{3 \pi} j_{\mathrm{c}} d_{\mathrm{fil}}\left(1+\left(j_{\mathrm{s}} / j_{\mathrm{c}}\right)^{2}\right)\left|\dot{B}_{1}\right|$.

\subsection{Parallel field}

If the applied field is purely parallel with the filaments then $B_{\mathrm{p} \|}=\mu_{0} j_{\mathrm{a}} d_{\mathrm{fil}} / 2$ and $M_{\mathrm{p} \|}=j_{\mathrm{a}} d_{\mathrm{fil}} / 6$, where $j_{\text {a }}$ refers to the critical current density in azimuthal direction. The loss for fully penetrated filaments is similar to eq. (1):

$p_{\mathrm{h} \|}=-M_{\mathrm{p} \|} \dot{B}_{\|}=\frac{j_{\mathrm{a}} d_{\mathrm{fil}}}{6}\left|\dot{B}_{\|}\right|$.

No thcory exists for calculating the hysteresis loss in the general case of a field change from $B$ to $B+\Delta B$. One approach is assume that the loss is determined entirely by the vector $\Delta \boldsymbol{B}$. The magnitude of the average field $|\boldsymbol{B}+\Delta \boldsymbol{B} / 2|$ is then only used to compute the value of $j_{c}$, which is considered to be constant during the interval $\Delta t$. Thus the possibility of rotating field components is excluded. Suppose $\Delta \boldsymbol{B}$ has an angle $\alpha$ with the axis of the wire. Clearly, the summation of losses caused by the separate field components $B_{\perp}=|\Delta \boldsymbol{B}| \sin \alpha$ and $B_{\|}=|\Delta \boldsymbol{B}| \cos \alpha$ is too pessimistic because the magnetization currents in axial direction interfere with those in azimuthal direction. A better method is suggested by Eikelboom [4]. His measurements on $\mathrm{Nb}_{3} \mathrm{Sn}$ samples show that the hysteresis loss can be expressed as

$p_{\mathrm{h}} \approx\left(\sin ^{2} \alpha\right) p_{\mathrm{h} \perp}+\left(1-\sin ^{2} \alpha\right) p_{\mathrm{h} \|}$,

i.e. an interpolation between $p_{\mathrm{h} \perp}$ and $p_{\mathrm{h} \|}$, the purely transverse and longitudinal cases respectively. Note that Martinelli [5] found a quite different dependence for $\mathrm{NbTi}$ wires. In large cables, due to the twisting and/or braiding, the angle $\alpha$ varies significantly as we follow a particular strand along its path. Therefore, it is useful to define $\gamma$ as the average of $\sin ^{2} \alpha$ over the cross section of the cable. For a field change $|\Delta \boldsymbol{B}| \gg$ $B_{\mathrm{p}}$, the encrgy loss per unit volume of filament becomes

$q_{\mathrm{h}} \approx|\Delta \boldsymbol{B}|\left(\gamma M_{\mathrm{p} \perp}+(1-\gamma) M_{\mathrm{p} \|}\right)$.

In the NET conductor the cabling angle of the strands varies between 0 and $60^{\circ}$, and the average is about $25^{\circ}$. So even $f$ the field is perpendicular to the cable, for example in the poloidal system, a substantial component parallel with the strands will be present.

\subsection{Restrictions}

As mentioned, eqs. (3) and (4) are based on the assumption that filaments remain fully penetrated by the external field. This is sufficiently accurate for the poloidal and toroidal coils of NET where the magnetic field will change several teslas during the operating cycle, while $B_{\mathrm{p} \perp}$ and $B_{\mathrm{p} \|}$ vary between 10 and $100 \mathrm{mT}$, depending on the actual magnitude of $\boldsymbol{B}$. If the amplitude of the changing field is of the order of $B_{\mathrm{p} \perp}$ and $B_{\mathrm{p} \|}$, it is necessary to modify eqs. (3) and (4). Again, the losses can be characterized by the wire parameters $j_{\mathrm{c}} d_{\mathrm{ril}}$ and $j_{\mathrm{a}} d_{\mathrm{fil}}$.

Magnetization or low-frequency loss measurements are required to determine the values of $j_{\mathrm{c}} d_{\mathrm{fil}}$ and $j_{\mathrm{i}} d_{\mathrm{fil}}$ as a function of the field because direct measurement of the azimuthal critical current density $j_{\mathrm{a}}$ is not possible. Furthermore, in some conductors the filaments show a collective behaviour so that the real diameter $d_{\text {fil }}$ is not representative for the magnetization anymore. In that case, an effective diameter $d_{\text {eff }}$ is often introduced, based on magnetization experiments. Approximate loss calculations are possible by replacing $d_{\mathrm{fil}}$ by $d_{\mathrm{eff}}$ in the formulac. The main cause for a difference between $d_{\text {fil }}$ and $d_{\text {eff }}$ is either coupling of the filaments due to the proximity effect, or actual bridging of filaments duc to the production process (for example in jelly-roll types of wires). Note that bridging or extreme variations of the filament cross section result in a non-uniform magnetization of the wire, preventing the definition of unique values of $B_{\mathrm{p}}$ and $M_{\mathrm{p}}$ that fully describe the losses.

\section{Coupling loss in multifilamentary wires}

Changing magnetic fields induce currents which flow along the outer filaments of the filamentary zone. The return currents flow through the matrix, transversely to 
the filaments, and cause dissipation. The preferred method for calculating this coupling current loss is to use Carr's continuum model [6] and solve Maxwell's equations

$$
\left\{\begin{array}{l}
\boldsymbol{\nabla} \times \boldsymbol{E}=-\partial_{\mathrm{t}} \boldsymbol{B}, \\
\nabla \times\left(\boldsymbol{B}-\mu_{0} \boldsymbol{M}\right)=\mu_{0} j .
\end{array}\right.
$$

The term $\mu_{0} \nabla \times \boldsymbol{M}$ can usually be neglected. Notice that solutions of these equations automatically satisfy the continuity equations $\boldsymbol{\nabla} \cdot \boldsymbol{j}=\mathbf{0}$ and $\boldsymbol{\nabla} \cdot \boldsymbol{B}=0$. Due to the twisted multifilamentary structure of the conductor the constitutive equation relating $j$ to $E$ is anisotropic

$$
\left\{\begin{aligned}
j_{\mathrm{r}}= & \sigma_{\perp} E_{\mathrm{r}} \\
j_{\varphi}= & \left(\sigma_{\perp}+\Delta \sigma \sin ^{2} \psi\right) E_{\varphi}+\Delta \sigma \sin \psi \cos \psi E_{z} \\
& +\eta j_{\mathrm{S}} \sin \psi, \\
j_{z}= & \left(\sigma_{\|}-\Delta \sigma \sin ^{2} \psi\right) E_{z}+\Delta \sigma \sin \psi \cos \psi E_{\varphi} \\
& +\eta j_{\mathrm{s}} \cos \psi,
\end{aligned}\right.
$$

with $\Delta \sigma=\sigma_{\|}-\sigma_{\perp}$ and $\psi=\operatorname{atan}\left(2 \pi r / L_{p}\right)$. Here, $L_{\mathrm{p}}$ represents the twist length, $\psi$ the local twist angle with respect to the axis, $\eta$ the relative fraction of superconductor, and $\eta j_{\mathrm{s}}$ the superconducting current density in the direction of the filaments. The conductivity of the composite is $\sigma_{\|}$along the filaments and $\sigma_{\perp}$ in perpendicular direction. The constitutive equation is non-linear because $j_{\mathrm{s}}$ depends non-linearly on the electrical field $E_{\|}$, as described by eq. (2). Due to the non-linearity we obtain moving boundaries between unsaturated regions where $\left|j_{\mathrm{s}}\right|<j_{\mathrm{c}}$ and saturated regions where $\left|j_{\mathrm{s}}\right|=j_{\mathrm{c}}$. Of course, in isotropic normal conducting regions (e.g. stabilizer material) eq. (8) reduces to the $\boldsymbol{j}=\boldsymbol{\sigma} \boldsymbol{E}$ because $j_{\mathrm{s}}=0$ and $\Delta \boldsymbol{\sigma}=\mathbf{0}$. After solving the system eqs. (2), (7) and (8), the local dissipation can be calculated as $\boldsymbol{E} \cdot \boldsymbol{j}-\mathbf{M} \cdot \partial_{\mathrm{t}} \boldsymbol{B}$.

A few authors $[7,8]$ have solved these equations numerically under the most general conditions. The results are very important for the insight in the current distribution and the actual processes inside the wire, but in view of the required computing time it is not practical for design purposes. Analytic solutions can be obtained under simplifying assumptions. For example, neglecting the thickness of the saturated regions and assuming that $d_{\text {fil }} \approx 0$ so that $E^{*}=0$.

For ramped transverse fields, it is customary to write the coupling loss per unit volume of conductor as

$p_{\mathrm{c}}=\tau_{\mathrm{c}} \frac{2}{\mu_{0}} \dot{B}_{\perp}^{2}$.

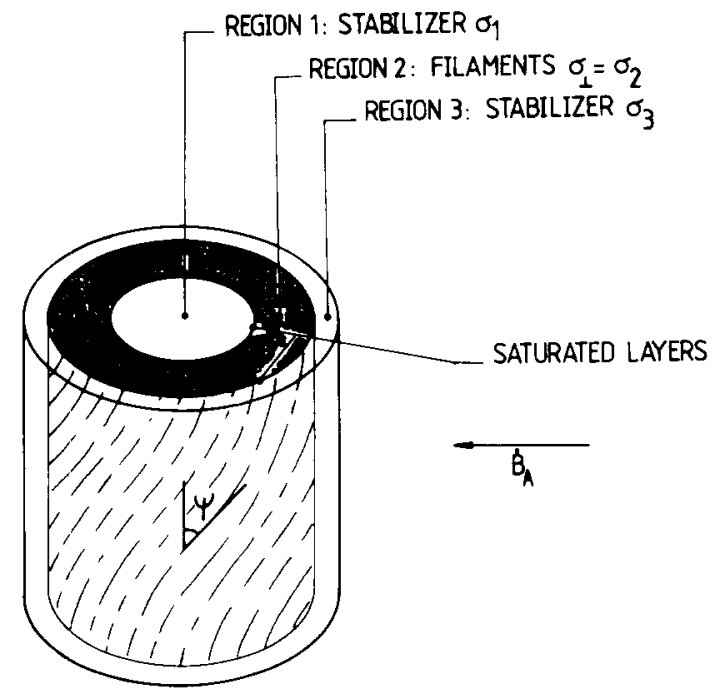

Fig. 1. Lay-out of a multifilamentary conductor having a central core and an outer region of stabilizer.

The constant $\tau_{c}$ can be attributed to the decay time of the coupling currents inside the composite, although several time constants may be involved if the wire has a complex geometry. The simple geometry, with uniformly distributed filaments leads to $[4,9]$

$\tau_{\mathrm{c}}=\frac{\mu_{0} \sigma_{\perp}}{2}\left(\frac{L_{\mathrm{p}}}{2 \pi}\right)^{2}\left(1+\left(\frac{2 \pi R}{L_{\mathrm{p}}}\right)^{2}\right)$

The extra factor $1+\left(2 \pi R / L_{\mathrm{p}}\right)^{2}$, caused by the $z$-component of the electrical field, is usually omitted in literature. In practice, wires often have a lay-out as shown in fig. 1, with several layers of different materials. We shall consider a wire that is subdivided in three regions, occupying the respective fractions $U_{1}, U_{2}$ and $U_{3}$ of the cross section (with $U_{1}+U_{2}+U_{3}=1$ ). Region 1 is the central core containing normal material of conductivity $\sigma_{1}$. Region 2 is the filamentary zone with transverse conductivity $\sigma_{2}$ and region 3 contains normal material with $\sigma_{3}$. Hartmann [3] solves Maxwell's equations for such a wire in a transverse field with constant $\dot{B}$. He also calculates the real shapes of the saturated regions that appear at the inner and outer radii of the filamentary zone. At low ramp rates these 


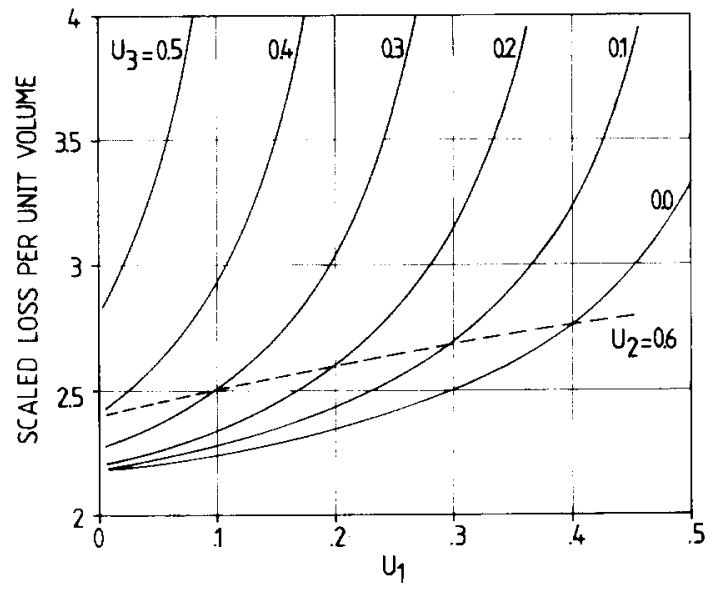

Fig. 2. Loss power density scaled by $\sigma_{1}(\dot{B} / \beta)^{2}$, for a threelayer conductor.

regions hardly contribute to the total dissipation and the losses per unit volume of wire become

$$
\begin{aligned}
& p_{1}=\sigma_{1}(\dot{B} / \beta)^{2} U_{1}\left[1+(\beta R / 2)^{2} U_{1}\right], \\
& p_{2}=\sigma_{2}(\dot{B} / \beta)^{2} U_{2}\left[1+(\beta R / 2)^{2}\left(2 U_{1}+U_{2}\right)\right], \\
& p_{3}=\sigma_{3}(\dot{B} / \beta)^{2} U_{3}\left[\left(U_{1}+U_{2}\right) /\left(1+U_{1}+U_{2}\right)\right. \\
& \left.+(\beta R / 2)^{2}\left(1+U_{1}+U_{2}\right)\right],
\end{aligned}
$$

with $\beta=2 \pi / L_{\mathrm{p}}$.

In many wires the regions 1 and 3 consist of copper while region 2 contains the filaments in copper matrix. An interesting question is where the filaments should be placed, in other words what are the optimum values of $U_{1}$ and $U_{3}$ if the overall fraction of superconductor $\eta_{\mathrm{sc}}$ is kept constant? The conductivity $\sigma_{2}$ is given by $\sigma_{1}\left(1+\eta_{2}\right) /\left(1-\eta_{2}\right)$, where $\eta_{2}$ refers to the local fraction of superconductor, equal to $\eta_{\text {sc }} / U_{2}$. Clearly, the values of $U_{1}$ and $U_{3}$ should not be large because this increases the conductivity in the filamentary region and thus the loss. Figure 2 shows such an analysis for $\eta_{\mathrm{sc}}=0.33$ and a twist pitch of 10 times the wire diameter. The total dissipation in the wire is scaled by the constant $\sigma_{1}(\dot{B} / \beta)^{2}$. The location of the filaments is not very critical but in principle the optimum solution is to distribute them homogeneously over the cross section. Also, at a given value of $U_{2}$ we observe an optimum when removing the central core and placing all stabilizer in the outer shell. In the case of a resistive barrier at the surface of the filaments the conductivity in region 2 is $\sigma_{1}\left(1-\eta_{2}\right) /\left(1+\eta_{2}\right)$, leading to a similar conclusion: the central core increases the coupling loss.
The above method was extended for conductors with more than 3 layers and formulated in a computer code which also takes into account the effect of a mixed matrix. In a mixed matrix region all materials are normal $\left(j_{\mathrm{s}}=0\right)$, but there is a large anisotropy, i.e. $\sigma_{\|} \gg \sigma_{\perp}$. Therefore, too optimistic loss figures will be found by just substituting the transverse conductivity $\sigma_{\perp}$ in formulas as eq. (11). Instead, we should integrate the local loss power density

$$
\begin{aligned}
p_{\mathrm{c}}= & \sigma_{\perp}\left(E_{r}^{2}+E_{\varphi}^{2}+E_{z}^{2}\right) \\
& +\left(\sigma_{\|}-\sigma_{\perp}\right)\left(E_{\varphi} \sin \psi+E_{z} \cos \psi\right)^{2} .
\end{aligned}
$$

Changing fields parallel with the conductor will also cause coupling losses. In a tokamak such parallel field variations mainly occur in the toroidal coils during a plasma disruption. But as mentioned, the conductors in the poloidal coils also feel a parallel field component due to the cabling angle. The longitudinal loss is a rather complicated subject and beyond the scope of this paper. The reader is referred to ref. [9].

\section{Interstrand coupling currents}

When several strands are cabled, the effective time constant, i.e. the loss per unit volume of composite, will increase. This is caused by interstrand coupling currents. The changing external field induces a complicated pattern of currents in the strands, with the essential feature that the current paths are closed via the cross-over contacts between the strands. At low ramp rates all currents are proportional to $\dot{B}$, although the actual magnitude and direction of the current depends on the position in the cable. As a result, if the electrical resistance of the cross-overs is constant and given by $R_{\mathrm{c}}$, the power loss is linear with $\dot{B}^{2} / R_{\mathrm{c}}$. In the NET prototype cables, the strands are plated with 2 $\mu \mathrm{m}$ chrome in order to enhance $R_{\mathrm{c}}$.

In a cable the anisotropy is not smooth, especially in a braided cable where adjacent strands can have completely different directions. Therefore, it is not appropriate to use a continuum model. A better method is to use a network of strands and set up Kirchhoff's equations for the various possible current paths. The characteristics of the superconductor are described by a non-linear relation between $I$ and $V$ for a finite element of strand with length $\Delta l$ :

$I= \begin{cases}I_{\mathrm{c}}(B) V / V^{*} & \text { if }|V|<V^{*}, \\ I_{\mathrm{c}}(B) \operatorname{sign}(V) & \text { if }|V|>V^{*},\end{cases}$ 


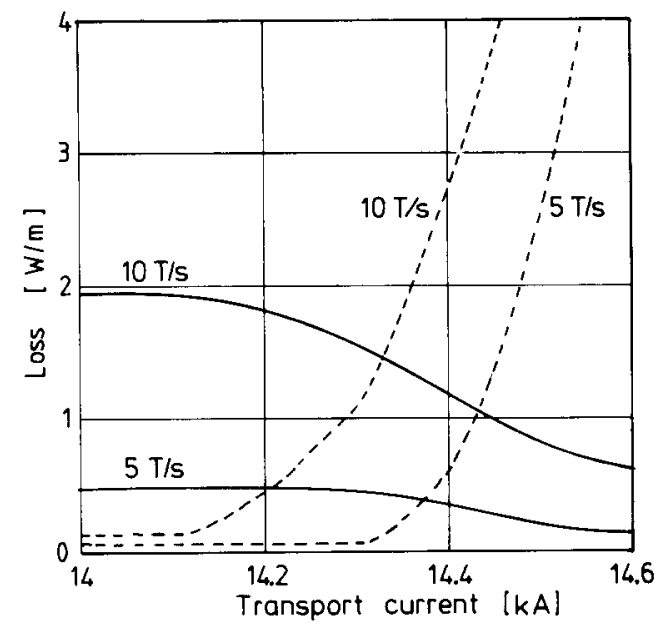

Fig. 3. Calculated dissipation in a braid for $5 \mathrm{~T} / \mathrm{s}$ and $10 \mathrm{~T} / \mathrm{s}$ using a network model. Solid lines represent the loss in the cross-overs. Dashed lines correspond to the transport current loss.

with $V^{*}=(4 / 3 \pi) \Delta l d_{\text {fil }}\left|\dot{B}_{\perp}\right|$. Note the similarity with the constitutive equation $j(E)$ in the continuum model. A network approach like this was used to calculate the interstrand loss in a 29-strands lattice braid [10], representing one subcable of a prototype cable for NET. The modelling consists of tracing the positions where the strands touch and finding the value $R_{\mathrm{c}}$. Of course, this description is approximate due to the irregularities in the geometry of the cable and the variations in the cross-over resistance $R_{\mathrm{c}}$. Some of the results are shown in fig. 3 and lead to the following conclusions:

- for low ramp rates the main dissipation occurs in the contact resistances $R$, i.e. very locally;

- the loss is then given by $\left(c_{x} B_{x}^{2}+c_{y} B_{y}^{2}+c_{z} B_{z}^{2}\right) / R_{c}$, where $c_{x}, c_{y}$ and $c_{z}$ depend on the geometry and can be converted into effective time constants;

- initially, the transport current loss in the strands is low, but when the sum of transport and coupling currents approaches $I_{\mathrm{c}}$ a sharp increase is observed. A similar analysis will be performed for a twisted cable. So far, experiments indicate that the ac loss in a braided cable is slightly lower than in a comparable cable made by twisting.

Since the loss depends on $R_{\mathrm{c}}$, it also depends on the Lorentz force acting on the cross-overs. The influence of the force on $R_{\mathrm{c}}$ was investigated experimentally at $4.2 \mathrm{~K}$ for two reacted $0.96 \mathrm{~mm}$ strands crossing at $90^{\circ}$. The experiment was repeated for different combinations with and without chrome plating, see fig. 4. Hysteresis is observed due to the plastic deformation

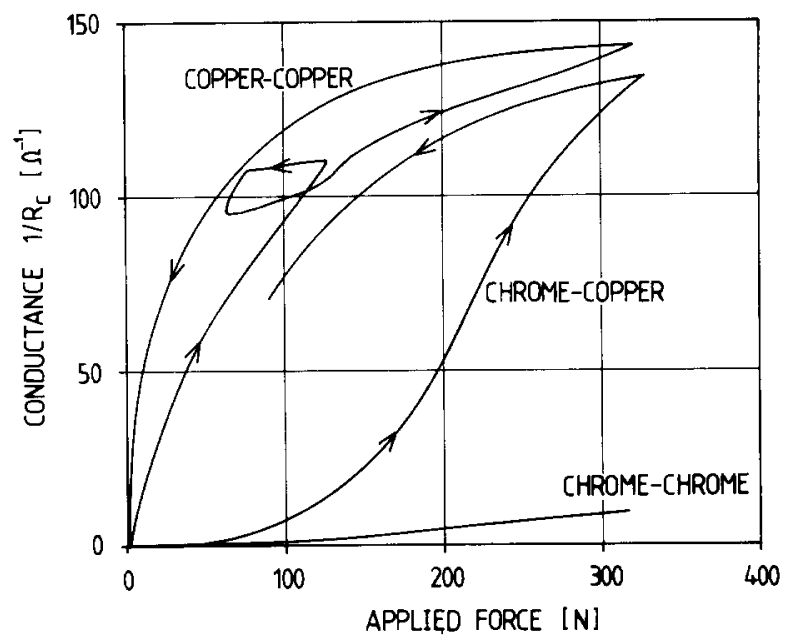

Fig. 4. Conductance $\left(1 / R_{\mathrm{c}}\right)$ versus force at $4.2 \mathrm{~K}$ for two strands crossing at $90^{\circ}$.

of the wires and after a $300 \mathrm{~N}$ test the deformed contact area shows up as a round imprint of half the wire diameter. The difference between the $\mathrm{Cr}-\mathrm{Cr}$ and $\mathrm{Cu}-\mathrm{Cu}$ combinations demonstrates how effective the 2 $\mu \mathrm{m}$ chrome plating is. The shape of the $\mathrm{Cr}-\mathrm{Cu}$ curve indicates that not only the high resistivity of the chrome is important, but also the mechanical properties which keep the contact area small. It should be noted that the compaction of the NET conductor into the jacket leads to more severe deformations and therefore lower contact resistances than in the above experiment.

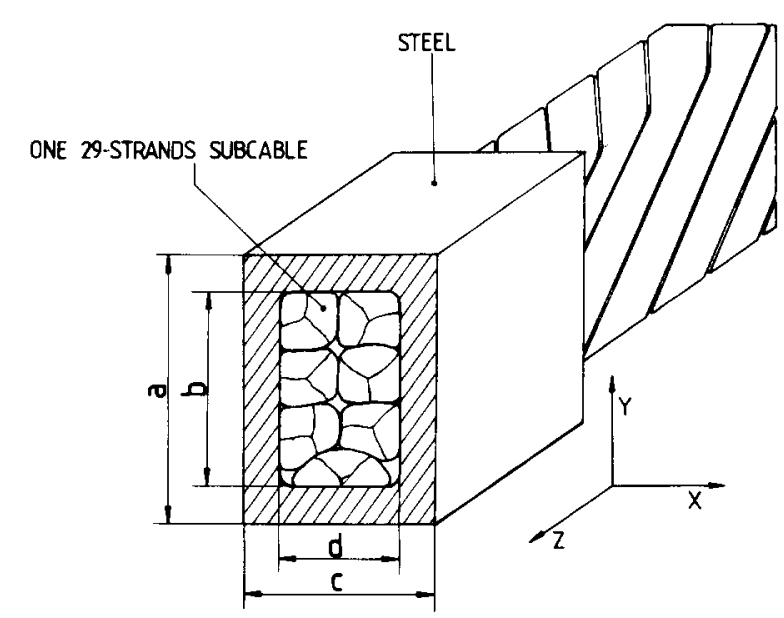

Fig. 5. Schematic view of a prototype cable-in-conduit for NET. 


\section{Influence of the jacket}

In the coil system, even the fastest variations of $B$ take place on a time scale which is much slower than the time constant of the steel jacket (in the order of $100 \mu \mathrm{s})$. Therefore, the shielding effect of the eddy currents in the jacket is negligible. The eddy-current loss per unit length of jacket is [11]

$p_{\mathrm{j}}=\sigma\left(\dot{B}_{x}^{2} J_{\mathrm{M} X}+\dot{B}_{y}^{2} J_{\mathrm{M} Y}+\dot{B}_{z}^{2} \frac{J_{\mathrm{M} X} J_{\mathrm{M} Y}}{J_{\mathrm{M} X}+J_{\mathrm{M} Y}}\right)$,

where $J_{M X}$ and $J_{M Y}$ are the moments of inertia about the $X$ - and $Y$-axis respectively. Using the dimensions $a$, $b, c$ and $d$ explained in fig. 5.

$J_{\mathrm{M} X}=\frac{c^{3} a-d^{3} b}{12}$ and $J_{\mathrm{MY}}=\frac{a^{3} c-b^{3} d}{12}$.

Figure 6 shows the results of loss measurements on different $35 \mathrm{~cm}$ long samples of a full-size prototype cable for NET. Clearly, the eddy current loss in the jacket is negligible as was predicted by calculation. Nevertheless, a significant difference can be observed between the losses of samples with and without jacket. The first explanation is that certain coupling currents exist that find their return path via the steel. In principle it is a source for extra losses, but calculations [12] shows that the resistance of the jacket is too high to account for the difference. The other explanation is

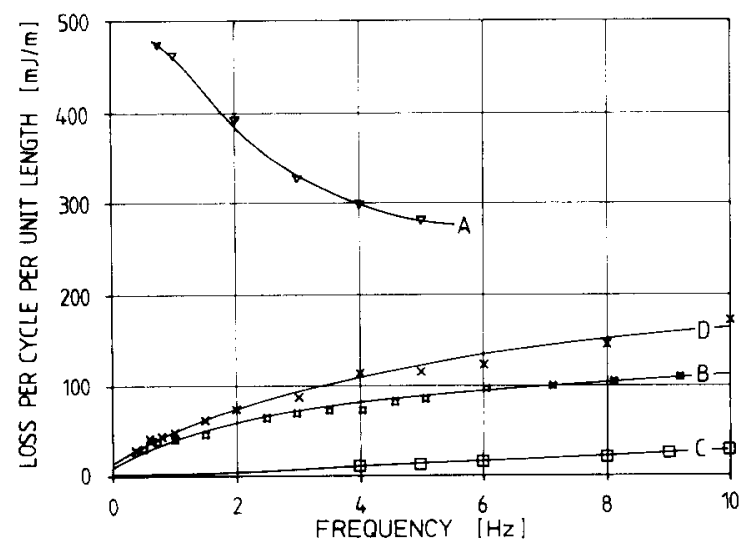

Fig. 6. Measured AC loss for $35 \mathrm{~cm}$ long NET samples in an alternating field of $25 \mathrm{mT}$ amplitude in $x$-direction; (a) complete cable, (b) conductor part only, (c) jacket only, (d) conductor insulated from the jacket. that the contact resistance $R_{\mathrm{c}}$ is reduced by removing the jacket. To test this idea, sample (b) was wrapped in $25 \mu \mathrm{m}$ Kapton insulation and reinserted in two halves of the jacket. The measured losses of this new sample are well below curve (a), but they confirm that the compressive force on the two halves has a strong influence on the loss.

\section{Combination of transport current and field changes}

Similar to eq. (3), a factor $1+\left(I / I_{\mathrm{c}}\right)^{2}$ is often used for wires and cables in order to correct the ac loss for the presence of a transport current. To our opinion this approach is very doubtful because of the current distribution inside the conductor and the fact that saturated regions will occur. In a wire for example, filaments passing through a saturated layer are no longer available for low-loss transport current [3]. Also in cables a sharp increase of the ac loss is theoretically predicted when the sum of transport current and coupling current in a strand approaches $I_{\mathrm{c}}$ [10]. This is equivalent to a reduction of the critical current. In a new test set-up at our institute such interactions between the different loss sources will be studied [13].

\section{Conclusions}

The ac loss of large superconducting cables consists of many contributions. Methods to compute these contributions were reviewed. The electrodynamic behaviour of the basic strand is well understood and its loss can be calculated with sufficient reliability, although the exact influence of fields parallel with the conductor requires extra study. By cabling the strands and compacting them into a jacket, extra loss sources are introduced which are complicated by the geometry of the cable. One of them, the interstrand coupling loss of the last cabling stage, needs special attention because it is relatively large and furthermore it may reduce the current-carrying capacity of the cable.

\section{Acknowledgments}

The major contribution to the described investigations must be attributed to our dear professor Louis van de Klundert, who passed away unexpectedly in september 1991 . The study is partly carried out under contract with NET Garching. 


\section{References}

[1] N. Mitchell, L. Bottura, P. Bruzzone, et al., $\mathrm{Nb}_{3} \mathrm{Sn}$ conductor development programme for superconducting coils for the European fusion programme, IEEE Trans. Magn. 27 (1991) 2064-2067.

[2] M.N. Wilson, Superconducting magnets (Oxford University Press, New York, 1983).

[3] R.A. Hartmann, A contribution to the understanding of AC losses in composite superconductors, Thesis University of Twente, Enschede, 1989.

[4] J.A. Eikelboom, AC losses in prototype conductors for the NET toroidal field coils, Thesis University of Twente, Enschede, 1991.

[5] A.P. Martinelli and B. Turck, Some effects of field orientation on the magnetization of superconducting wires, Cryogenics 28 (1978) 155-161.

[6] W.J. Carr Jr, AC loss in a twisted filamentary superconducting wire, J. Appl. Phys. 45 (1974) 929-934.

[7] P.C. Rem, et al., A simulation of a multifilamentary wire carrying a transport current in an AC applied field, in: Proc. ICEC-11 (Butterworth Ltd, Guildford, 1986) pp. $751-755$
[8] A. Février, Losses in a twisted multifilamentary superconducting composite submitted to any space and time variations of the electromagnetic surrounding, Cryogenics 23 (1983) 185-200.

[9] L.J.M. van de Klundert, E.M.J. Niessen and P.J. Zandbergen, Electromagnetic response of composite superconducting wires, Journal of Engineering Mathematics 26 (1992) 231-265.

[10] E.M.J. Niessen and L.J.M. van de Klundert, A Numerical model for the 29-strand NET braid, IEEE Trans. Magn. 27 (1991) 1896-1899.

[11] K.V. Namjoshi and P.P. Biringer, Low frequency eddy current loss estimation in long conductors by using the moment of inertia of cross sections, IEEE Trans. Magn. 24 (1988) 2181-2185.

[12] A.J.M. Roovers, AC loss measurements on full-size cables for NET, internal report, 1991.

[13] G.B.J. Mulder, et al., A new test set-up to measure the AC losses of the conductors for NET, IEEE Trans. Magn. 27 (1991) 2190-2193. 